\title{
On Records in Sequences of Nonidentically Distributed Exponential Random Variables
}

\author{
M. Ahsanullah \\ Rider University, Lawrenceville, New Jersey, USA \\ V.B. Nevzorov \\ St. Petersburg State University, St. Petersburg, Russia
}

Received 1 November 2016

Accepted 1 March 2017

\begin{abstract}
The representation of record values based on sequences of non-identically distributed exponential random variables in terms of independent exponential summands is suggested.

Keywords: Record values; Record indicators; Exponential distributions
\end{abstract}

Let $\mathrm{Z}, \mathrm{Z}_{1}, \mathrm{Z}_{2}, \ldots$ be independent random variables (r.v.'s) having the standard exponential $\mathrm{E}(1)$ - distribution with distribution function (d.f.)

$$
\mathrm{F}(\mathrm{x})=\max \{0,1-\exp (-\mathrm{x})\} .
$$

We also will deal with exponentially $\mathrm{E}(\lambda)$ - distributed r.v.'s having d.f.'s

$$
\mathrm{F} \lambda(\mathrm{x})=\max \{0,1-\exp (-\mathrm{x} / \lambda)\}, \quad \lambda>0 .
$$

Let also $\mathrm{L}(1)<\mathrm{L}(2)<\ldots$ and $\mathrm{Z}(1)<\mathrm{Z}(2)<\ldots$ be the corresponding upper record times and upper record values. We introduce these r.v.'s by relations

$$
\mathrm{L}(1)=1, \mathrm{Z}(1)=\mathrm{Z}_{1} \quad \text { and } \quad \mathrm{L}(\mathrm{n})=\min \left\{\mathrm{j}: \mathrm{Z}_{\mathrm{j}}>\mathrm{Z}(\mathrm{n}-1)\right\}, \mathrm{Z}(\mathrm{n})=\mathrm{Z}_{\mathrm{L}(\mathrm{n})}, \mathrm{n}=2,3, \ldots
$$

As it is known, any exponential $\mathrm{E}(\lambda)$ - distribution possesses the so-called memory less property, when

$$
\mathrm{P}\{\mathrm{Z}>\mathrm{x}+\mathrm{y} \mid \mathrm{Z}>\mathrm{x}\}=\mathrm{P}\{\mathrm{Z}>\mathrm{y}\}, \mathrm{x}>0, \mathrm{y}>0 .
$$

Equality (1) allows to obtain very convenient relations for record values $Z(n), n=1,2, \ldots$. Among them we can mention the next result.

Representation 1. If $Z_{1}, Z_{2}, \ldots$ are independent $E(1)$-distributed r.v.'s then the following equalities in distribution are valid:

$$
\{\mathrm{Z}(1), \mathrm{Z}(2), \ldots, \mathrm{Z}(\mathrm{n})\} \stackrel{d}{=}\left\{\mathrm{Z}_{1}, \mathrm{Z}_{1}+\mathrm{Z}_{2}, \ldots, \mathrm{Z}_{1}+\mathrm{Z}_{2}+\ldots+\mathrm{Z}_{\mathrm{n}}\right\}, \mathrm{n}=1,2, \ldots
$$


Remark 1. It is easy to check that equalities (2) are also valid in the case when r.v.'s $Z_{1}, Z_{2}, \ldots$ have any common $\mathrm{E}(\lambda)$-distribution.

Together with record values $\mathrm{Z}(1)<\mathrm{Z}(2)<\ldots$ let us consider the record indicators $\xi_{\mathrm{n}}, \mathrm{n}=1,2, \ldots$, each of them takes value one, if the corresponding $Z_{n}$ is a record, and it is equal zero, otherwise. It is known (see, for example, monographs [1]-[6], where the record theory is presented), that these indicators are independent and $\mathrm{P}\left\{\xi_{\mathrm{n}}=1\right\}=1 / \mathrm{n}$. Indeed, this result stays valid for indicators of record values based on any sequence of independent r.v.'s having a common continuous distribution function $\mathrm{F}(\mathrm{x})$.

Now we try to understand, what type of relations, analogous to (2), can be obtained, if instead of identically distributed $Z_{1}, Z_{2}, \ldots$ one will deal with independent r.v.'s $Z_{k} \sim E\left(\lambda_{k}\right), k=1,2, \ldots$, having exponential distributions with different parameters $\lambda_{\mathrm{k}}>0$.

Let us now consider a sequence of independent r.v.'s $Z_{k} \sim E\left(\lambda_{k}\right), k=1,2, \ldots$, and their d.f.'s

$$
\mathrm{F}_{\mathrm{k}}(\mathrm{x})=\max \left\{0,1-\exp \left(-\mathrm{x} / \lambda_{\mathrm{k}}\right)\right\},-\infty<\mathrm{x}<\infty, \lambda_{\mathrm{k}}>0, \mathrm{k}=1,2, \ldots
$$

It appears that in this scheme one must deal with very complicated d.f.'s of record values $Z(n)$. Say, even in the simplest case $n=2$ we have the following equality:

$$
\mathrm{P}\{\mathrm{Z}(2)>\mathrm{x}\}=\frac{1}{\lambda_{1}} \sum_{k=2}^{\infty} \exp \left(-x / \lambda_{k}\right) \int_{0}^{x}\left(1-\exp \left(-\mathrm{u} / \lambda_{2}\right)\right) \ldots\left(1-\exp \left(-\mathrm{u} / \lambda_{k-1}\right)\right) \exp \left(-\mathrm{u} / \lambda_{1}\right) \mathrm{du} .
$$

Analogously, very complicate relations in this case are valid for record indicators $\xi_{n}, n=1,2, \ldots$ :

$$
\mathrm{P}\left\{\xi_{\mathrm{n}}=1\right\}=1-\mathrm{P}\left\{\xi_{\mathrm{n}}=0\right\}=\frac{1}{\lambda_{n}} \int_{0}^{\infty}\left(1-\exp \left(-\mathrm{u} / \lambda_{2}\right)\right) \ldots\left(1-\exp \left(-\mathrm{u} / \lambda_{n-1}\right)\right) \exp \left(-\mathrm{u} / \lambda_{n}\right) \mathrm{du}
$$

For example, we get from (5) that

$$
\mathrm{P}\left\{\xi_{2}=1\right\}=\lambda_{2} /\left(\lambda_{1}+\lambda_{2}\right)
$$

and

$$
\mathrm{P}\left\{\xi_{3}=1\right\}=1-\lambda_{1} /\left(\lambda_{1}+\lambda_{2}\right)-\lambda_{2} /\left(\lambda_{2}+\lambda_{3}\right)+\lambda_{1} \lambda_{2} /\left(\lambda_{1} \lambda_{2}+\lambda_{1} \lambda_{3}+\lambda_{2} \lambda_{3}\right) .
$$

Note also that

$$
\begin{aligned}
\mathrm{P}\left\{\xi_{2}=1, \xi_{3}=1\right\} & =\frac{1}{\lambda_{2}} \int_{0}^{\infty}\left(1-\exp \left(-\mathrm{u} / \lambda_{1}\right)\right) \exp \left(-\mathrm{u} / \lambda_{3}\right) \exp \left(-\mathrm{u} / \lambda_{2}\right) \mathrm{du} \\
& =\lambda_{3} /\left(\lambda_{2}+\lambda_{3}\right)-\lambda_{1} \lambda_{3} /\left(\lambda_{1} \lambda_{2}+\lambda_{1} \lambda_{3}+\lambda_{2} \lambda_{3}\right) .
\end{aligned}
$$

Taking into account the simplest situation with indicators $\xi_{2}$ and $\xi_{3}$ and considering the corresponding equalities (6)-(8), we get that the independence property of the record indicators is not saved in the general case, when parameters

$$
\lambda_{\mathrm{k}}, \mathrm{k}=1,2, \ldots, \text { are different. }
$$

The situation with non-identically distributed exponential r.v.'s will improved if to consider the following record scheme. 
Let now $Z_{1} \sim E\left(\lambda_{1}\right)$ and $L(1)=1$. The next r.v.'s $Z_{2}, Z_{3}, \ldots, Z_{L(2)}$ (up to the moment $L(2)$, when the first element in this sequence will exceed $Z_{1}$ ) have

$\mathrm{E}\left(\lambda_{2}\right)$-distribution. Then r.v.'s $\mathrm{Z}_{\mathrm{L}(2)+1}, \mathrm{Z}_{\mathrm{L}(2)+2, \ldots,} \mathrm{Z}_{\mathrm{L}(3)}$ are proposed to have

$\mathrm{E}\left(\lambda_{3}\right)$-distribution. Here L(3) is the first time when the first in this sequence random variable becomes more than $Z_{L(2)}$. Continuing the suggested process we introduce a new series $Z_{L(n)+1}, Z_{L(n)+2}, \ldots, Z_{L(n+1)}$ of $E\left(\lambda_{n+1}\right)-$ distributed r.v.'s each time when we get the record element in the previous series. Thus we deal with record values $Z(1)=Z_{1}, Z(2)=Z_{L(2)}, \ldots, Z(n)=Z_{L(n)}, \ldots$, based on series of independent non-identically distributed exponential r.v.'s.

For our convenience let us introduce one more sequence of independent $E(1)$ - distributed r.v.'s $\eta_{1}, \eta_{2}, \ldots$. The next result is valid.

Representation 2. The following equalities are valid for record values $Z(1)<Z(2)<\ldots$ defined above:

$$
\{Z(1), Z(2), \ldots, Z(n)\}^{d}=\left\{\lambda_{1} \eta_{1}, \lambda_{1} \eta_{1}+\lambda_{2} \eta_{2}, \ldots, \lambda_{1} \eta_{1}+\lambda_{2} \eta_{2}+\ldots+\lambda_{n} \eta_{n}\right\}, \quad n=1,2, \ldots,
$$

where $\eta_{1}, \eta_{2}, \ldots$ are independent exponentially E(1)- distributed r.v.'s.

Remark 2. One can see that inter-record values $Z(1), Z(2)-Z(1), \ldots, Z(n+1)-Z(n)$ in the suggested scheme are independent and have exponential distributions.

To prove the validity of relations (9) one can see that for any $0<\mathrm{y}<\mathrm{x}$ we get the following equality:

$$
\begin{aligned}
\mathrm{P}\{\mathrm{Z}(2)>\mathrm{x} \mid \mathrm{Z}(1)=\mathrm{y}\} & =\mathrm{P}\left\{\mathrm{Z}_{2}>\mathrm{x}\right\}+\mathrm{P}\left\{\mathrm{Z}_{2} \leq \mathrm{y}, \mathrm{Z}_{3}>\mathrm{x}\right\}+\mathrm{P}\left\{\mathrm{Z}_{2} \leq \mathrm{y}, \mathrm{Z}_{3} \leq \mathrm{y}, \mathrm{Z}_{4}>\mathrm{x}\right\}+\ldots \\
& =\exp \left(-\mathrm{x} / \lambda_{2}\right)+\left(1-\exp \left(-\mathrm{y} / \lambda_{2}\right)\right) \exp \left(-\mathrm{x} / \lambda_{2}\right)+\left(1-\exp \left(-\mathrm{y} / \lambda_{2}\right)\right)^{2} \exp \left(-\mathrm{x} / \lambda_{2}\right)+\ldots \\
& =\exp \left\{-(\mathrm{x}-\mathrm{y}) / \lambda_{2}\right\} .
\end{aligned}
$$

It means that

$$
\mathrm{Z}(2) \stackrel{d}{=} \mathrm{Z}(1)+\lambda_{2} \eta_{2}
$$

and $Z(1)$ and $\lambda_{2} \eta_{2}$ are independent summands in (11).

Analogously one gets that

$$
\mathrm{Z}(\mathrm{n}) \stackrel{d}{=} \mathrm{Z}(\mathrm{n}-1)+\lambda_{\mathrm{n}} \eta_{\mathrm{n}}, \mathrm{n}=3,4, \ldots,
$$

where $\mathrm{Z}(\mathrm{n}-1)$ and $\eta_{\mathrm{n}}$ are also independent r.v.'s.

Combining all these relations one gets the validity of relations (9).

Remark 3. Indeed, if to take $\lambda_{\mathrm{k}}=1, \mathrm{k}=1,2, \ldots$, in Representation 2, one gets, as a partial case, the result, given in Representation 1.

Remark 4. Thus we suggested the situation when it is possible to express distributions of records based on non-identically distributed exponential random variables via distributions of independent random summands. If to discuss record indicators in the given scheme it is necessary to mention that in the general case, when we deal with non-identically distributed r.v.'s $Z_{1}, Z_{2}, \ldots$, these indicators are dependent. To check this fact it is enough to mention that for any $n=3,4, \ldots$ probabilities $\mathrm{P}\left\{\xi_{2}=0, \xi_{3}=0, \ldots, \xi_{\mathrm{n}}=0\right\}$ depend on $\lambda_{1}$ and $\lambda_{2}$ only, while any probability $\mathrm{P}\left\{\xi_{\mathrm{k}}=0\right\}, \mathrm{k}=2,3, \ldots, \mathrm{n}$, depends on $\mathrm{k}$ parameters $\lambda_{1}, \lambda_{2}, \ldots, \lambda_{\mathrm{k}}$. 


\section{References}

[1] M. Ahsanullah, Record Statistics (Nova Science Publishers Inc., NY, NY, 1995).

[2] M. Ahsanullah, Record Values. Theory and Applications (University Press of America, Lanham, MD, 2004).

[3] M Ahsanullah and V.B. Nevzorov, Records via Probability Theory (Atlantic Press in Probability and Statistics. Atlantis Press, 2015).

[4] M. Ahsanullah and G.P. Yanev, (2008). Records and Branching Processes (Nova Science Publishers Inc., NY, NY, 2008).

[5] B.C. Arnold, N. Balakrishnan and H.N. Nagaraja, Records (John Wiley \& Sons Inc., NY, NY, 1998).

[6] V.B. Nevzorov , Records: Mathematical Theory Translations of Mathematical Monographs, Volume 194 (American Mathematical Society, Providence, RI 2000). 